\title{
Porosity and thickness characterization of porous Si and oxidized porous Si layers - an ultraviolet-visible-mid infrared ellipsometry study
}

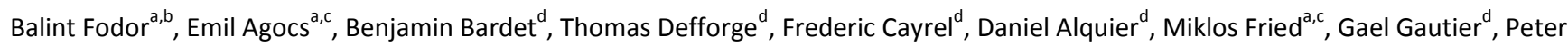
Petrik $^{\mathrm{a}, \mathrm{c}}$

anstitute for Technical Physics and Materials Science (MFA), Centre for Energy Research of the Hungarian Academy of Sciences, Konkoly Thege Str. 29-33, 1121 Budapest, Hungary

${ }^{b}$ Doctoral School of Physics, Faculty of Science, University of Pécs, 7624 Pécs, Ifjúság útja 6, Hungary

'Doctoral School of Molecular-and Nanotechnologies, Faculty of Information Technology, University of Pannonia, Egyetem u. 10, Veszprem, $\mathrm{H}$ 8200, Hungary

${ }^{d}$ Université François Rabelais de Tours, CNRS, CEA, INSA-CVL, GREMAN UMR 7347, Tours, France

*Corresponding author. Electronic address: fodor@mfa.kfki.hu $\quad+36203617876$

This paper suggests the evaluation of morphological parameters of porous silicon layers (PSL) using spectroscopic ellipsometry from UV to midinfrared optical range. PSL were prepared by electrochemical etching of monocrystalline silicon wafers in hydrofluoric acid-based electrolyte. Measuring with an optical and an infrared ellipsometer with a wide spectral range permits an accurate characterization of PSL properties from the top surface to the bottom of the layer with thicknesses from several hundred nanometers up to a few tens of micrometers. Several different optical models for ellipsometric evaluations were developed to determine the thickness, the average porosity, the in-depth porosity gradient, the oxidation level and the surface roughness of the PSL. Porosity was modeled with multiple effective medium layers by varying ratio of crystalline silicon, void and oxidized silicon wherever needed. Thin PSL $(<5 \mu \mathrm{m})$ shows no impact of current density on porosity and thickness. However, evaluation of thick PSL $(20-50 \mu \mathrm{m})$ highlights the in-depth porosity gradient. Thickness values were also cross-checked with electron microscopy confirming the proposed ellipsometric models. Additionally, different oxidation techniques have also been compared in terms of oxidation level and void content. Volume expansion during PSL oxidation follows exactly the same behavior as that during the oxidation of planar silicon wafers.

Keywords:

Infrared ellipsometry; Electrochemical etching; Porous silicon layer; Oxidation; Measurement of porosity. 


\section{Introduction}

Over the last few years, porous silicon layers (PSL) aroused enthusiasm for its many potential industrial applications. For instance, PSL can be used as sensors [1], as anodes in Lithium-ion battery [2], or in microelectronics thanks to its electrical insulating properties $[3,4]$. Both structural and chemical properties of PSL are key parameters in order to obtain the desired properties (Handbook of Porous Silicon, p. 202/203 [5]). Electrochemical parameters (current density, electrolyte composition...) allow a fine tuning of the micro- or nanostructure: porosity, pore size or specific surface area [6]. On the other hand, the control of the surface chemistry (through oxidation, carbonization or metallization) is also indispensable to functionalize and stabilize PSL with the expectation of a better reliability [7].

In order to mechanize PSL in an industrial process flow, there is a need to set up a fast, reliable and non-destructive control of PSL characteristics. Non-destructive porosity measurement techniques can generally be sorted depending on PSL thickness. For ultrathin layers (less than $300 \mathrm{~nm}$ ), X-ray techniques can be used such as X-ray reflectometry [8] or grazing-incidence smallangle X-ray scattering [9]. These techniques provide an accurate measurement of the very superficial layer. When it comes to thicker layers, infrared spectroscopy is the most frequently used technique in the form of Fourier transform infrared spectroscopy (one reflectance spectrum) $[10,11]$ or spectroscopic liquid infiltration method (two reflectance spectra) [12].

Spectroscopic ellipsometry (SE) is a very powerful optical technique when it comes to thin layers, surface or near-surface characterization, as it is a fast - typically below one second for a full ultraviolet-near infrared $(191-1690 \mathrm{~nm})$ spectrum -, labelfree and non-destructive measurement technique, in addition to being very sensitive to the optical properties probed by the polarized light [13]. Recently, it has also been demonstrated for inline analysis of roll-to-roll thin-film photovoltaics with a novel divergent illumination mapping setup [14]. SE measurement with illumination in the visible, ultraviolet and near infrared range has a great potential to sensitively characterize PSL up to a few $\mu$ m thick layers [15-17]. However, for larger thicknesses, interference oscillations become too dense to be accurately resolved with multichannel detection systems. Of course, measurements with high-resolution monochromators could attain a better result, and thus thicker layer investigations would be possible (around $10 \mu \mathrm{m}$ thickness). However, a wide spectral range measurement, which is necessary for accurate porosity content and porosity distribution determination, in this way would take orders of magnitude more time ( $\sim$ one day) than multichannel measurements. To surpass this limitation, mid-infrared ellipsometric measurements performed on the porous samples allow the characterization of up to several tens of micrometers in thickness. Additionally, with visible ellipsometry it is difficult to separate the optical response of the oxide content from the porosity thus diminishing individual sensitivity for both [18-20]. However, with mid-infrared ellipsometry, where this optical separation (in refractive index and extinction coefficient) is enhanced, due to the molecular resonance peaks of silicon dioxide [21], sensitivity is much greater for quantitative analysis of the oxidation level. In this study, both visible and mid-infrared measurements were performed on the samples to combine their advantages such as surface sensitivity of visible and composition sensitivity of infrared ellipsometry, and to be able to investigate PSL covering a wide thickness range. Different optical models for ellipsometric evaluations were developed with increasing complexity to achieve best fit on the measured spectra while maintaining low fit error and low parameter cross-correlations, and thus obtaining physically plausible and relevant information about the samples. To summarize, these relevant information include: porous layer average thickness, thickness non-uniformity, average porosity, indepth porosity gradient, oxidation level and surface roughness.

\section{Experimental details}

2.1. PSL etching conditions

Porous silicon is obtained by electrochemical etching of a monocrystalline silicon wafer in a $30 \mathrm{wt}$. \% hydrofluoric acid (HF) acetic acid ( 25 wt. \%) solution. This work focuses on mesoporous silicon, i.e. with pore diameters in the range of $2-50 \mathrm{~nm}$ [22], obtained from (100)-oriented highly-doped n-type (0.01-0.015 $\Omega . c m)$ silicon. By changing anodization current density and duration, both thickness and porosity of PSL can be adjusted. After electrochemical etching, PSL were carefully rinsed with deionized water and dried on a hotplate $\left(120^{\circ} \mathrm{C}\right)$ to remove the remaining electrolyte from the pores. Table 1 summarizes the electrochemical conditions of the different PSL investigated in the present work. The respective dimensions of thin layers (samples $\mathrm{N}-01$ to $\mathrm{N}-08$ ) are determined thanks to UV-visible ellipsometer and are distinguished from thicker layers (samples N-09 to N-11) whose characteristics are measured by infrared ellipsometry. The PSL thicknesses have also been determined by scanning electron microscopy (SEM). Figs. $1 \mathrm{a}$ and $1 \mathrm{~b}$ show samples with PSL thicknesses of $3.3 \mu \mathrm{m}(\mathrm{N}-08)$ and $52 \mu \mathrm{m}(\mathrm{N}-11)$, respectively, focused at the silicon/porous silicon interface.

TABLE 1: Electrochemical etching conditions and thickness measurement values of the investigated porous silicon layers.

\begin{tabular}{|c|r|r|r|r|}
\hline Sample & $\begin{array}{c}\text { Current } \\
\text { density } \\
\left(\mathrm{mA} / \mathrm{cm}^{2}\right)\end{array}$ & $\begin{array}{c}\text { Etching } \\
\text { duration } \\
(\mathrm{s})\end{array}$ & $\begin{array}{c}\text { Thickness } \\
\text { by SE* } \\
(\mu \mathrm{m})\end{array}$ & \multicolumn{2}{|c|}{$\begin{array}{c}\text { Thickness } \\
\text { by SEM } \\
(\mu \mathrm{m})\end{array}$} \\
\hline $\mathrm{N}-01$ & 14.5 & 15 & 0.7 & 0.7 \\
\hline $\mathrm{N}-02$ & 14.5 & 30 & 1.2 & 1.3 \\
\hline $\mathrm{N}-03$ & 14.5 & 60 & 2.4 & 2.3 \\
\hline $\mathrm{N}-04$ & 14.5 & 120 & 4.2 & 4.4 \\
\hline $\mathrm{N}-05$ & 116.3 & 2 & 0.7 & 0.7 \\
\hline $\mathrm{N}-06$ & 116.3 & 4 & 1.2 & 1.3 \\
\hline $\mathrm{N}-07$ & 116.3 & 6 & 1.8 & 1.8 \\
\hline $\mathrm{N}-08$ & 116.3 & 12 & 3.3 & 3.4 \\
\hline \hline $\mathrm{N}-09$ & 80.0 & 210 & 23.0 & 25.0 \\
\hline $\mathrm{N}-10$ & 80.0 & 300 & 31.3 & 31.0 \\
\hline $\mathrm{N}-11$ & 80.0 & 600 & 52.0 & 52.0 \\
\hline
\end{tabular}

*Thickness by SE values for $\mathrm{N}-01-\mathrm{N}-08$ are averages from UV-NIR and MIR fit results, while for $\mathrm{N}-09-\mathrm{N}-11$ they are obtained solely from MIR fit results. 

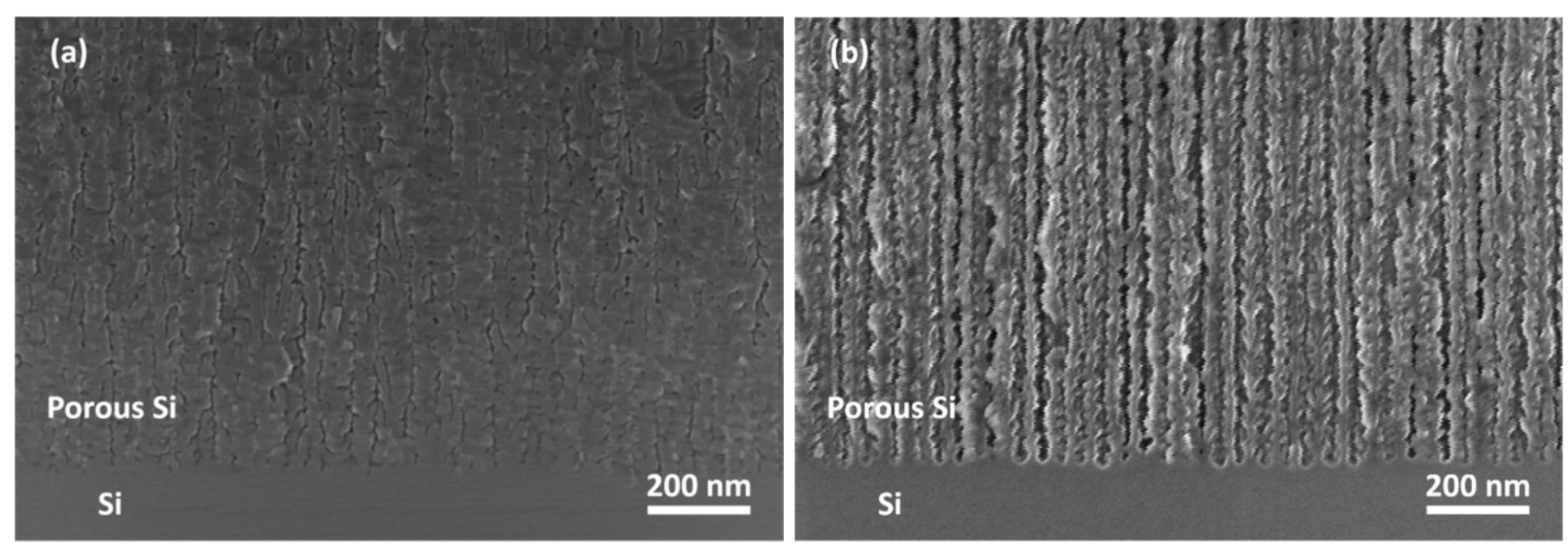

Figure 1: SEM images of (a) sample N-08 and (b) N-11 focused the silicon/porous silicon interface.

\subsection{PSL composition determination}

It is well-known that PSL can be oxidized by various methods, including thermal oxidation (wet or dry), chemical oxidation or electrochemical oxidation (Handbook of Porous Silicon, p. 1-24 [23]). $p^{+}$-type $(0.02 \Omega . c m)$ samples were used to study the chemical composition of PSL after oxidation. A 6-inch silicon wafer was anodized with the same electrochemical conditions as sample $\mathrm{N}-10$, and then cut in pieces. Oxidation was performed either by dry thermal oxidation in an $\mathrm{O}_{2}$-rich atmosphere in a tubular furnace or by electrochemical oxidation in an aqueous solution composed of 10 wt. \% acetic acid. After electrochemical oxidation, the oxidized PSL were rinsed with deionized water and dried on hotplate $\left(120^{\circ} \mathrm{C}\right)$. To improve the density of the electrochemical oxide, a high-temperature annealing post-treatment is usually performed [24]. In the present study, densification annealing was carried out with the same recipe as thermal oxidation reference. Table 2 summarizes the different oxidation conditions investigated in the present work.

TABLE 2: List of investigated oxidized samples with oxidation technique and experimental condition descriptions.

\begin{tabular}{|c|c|c|}
\hline $\begin{array}{l}\text { Sample } \\
\text { notation }\end{array}$ & $\begin{array}{l}\text { Oxidation } \\
\text { technique }\end{array}$ & Experimental conditions \\
\hline Ox-ref & $\begin{array}{l}\text { Reference sample } \\
\text { without any post- } \\
\text { treatments }\end{array}$ & $\begin{array}{l}\text { Anodization in } 30-w t . \% \mathrm{HF} \text { and } 25- \\
\text { wt.\% acetic acid, using a current } \\
\text { density of } 80 \mathrm{~mA} / \mathrm{cm}^{2} \text { for } 300 \mathrm{~s} \text {. }\end{array}$ \\
\hline Ox-1 & Pre-oxidized sample & $\begin{array}{l}\text { Ox-ref }+ \text { thermal oxidation at } 300^{\circ} \mathrm{C} \\
\text { in } \mathrm{O}_{2} \text { for } 1 \mathrm{~h}\end{array}$ \\
\hline $0 x-2$ & $\begin{array}{l}\text { Electrochemically } \\
\text { oxidized sample }\end{array}$ & $\begin{array}{l}\text { Ox-ref }+ \text { anodic oxidation ( } 10 \\
\mathrm{~mA} / \mathrm{cm}^{2} \text { for } 0.5 \mathrm{~h} \text { ) in } 10-\mathrm{wt} . \% \text { acetic } \\
\text { acid }\end{array}$ \\
\hline $0 x-3$ & $\begin{array}{l}\text { Densified } \\
\text { electrochemically } \\
\text { oxidized sample }\end{array}$ & $\begin{array}{l}\text { Ox-2 + thermal oxidation at } 800^{\circ} \mathrm{C} \text { in } \\
\qquad \mathrm{O}_{2} \text { for } 1 \mathrm{~h}\end{array}$ \\
\hline Ox-4 & $\begin{array}{l}\text { Thermal oxidation } \\
\text { for } 30 \mathrm{~min}\end{array}$ & $\begin{array}{l}\text { Ox-ref }+ \text { thermal oxidation at } 800^{\circ} \mathrm{C} \\
\text { in } \mathrm{O}_{2} \text { for } 0.5 \mathrm{~h}\end{array}$ \\
\hline $0 x-5$ & $\begin{array}{l}\text { Thermal oxidation } \\
\text { for } 60 \mathrm{~min}\end{array}$ & $\begin{array}{l}\text { Ox-ref + thermal oxidation at } 800^{\circ} \mathrm{C} \\
\text { in } \mathrm{O}_{2} \text { for } 1 \mathrm{~h}\end{array}$ \\
\hline
\end{tabular}

\subsection{SE measurement setup}

The SE measurements performed on the PSL samples were made with an optical (ultraviolet-near-infrared, UV-NIR) and a mid-infrared (MIR) ellipsometer allowing evaluations in a wide spectral range. The UV-NIR measurements were performed with a Woollam M-2000DI variable angle spectroscopic ellipsometer. It utilizes the rotating compensator technology with two multichannel detection systems. This setup enables the measurement of both ellipsometric angles ( $\Psi$ and $\Delta$, the amplitude ratio and phase difference, respectively, of the complex reflection coefficients of light polarized parallel and perpendicular to the plane of incidence $[25,26]$ ) with an accuracy of $5 \times 10^{-2}$ in the wavelength range from 191 to $1690 \mathrm{~nm}(6.49-0.73 \mathrm{eV})$, with a spectral resolution of about $1.5 \mathrm{~nm}$ in the lower (short wavelength) part and about $3.5 \mathrm{~nm}$ in the upper part of the spectrum, totaling in 706 wavelength points. The angles of incidence of the illumination were chosen to be between $65^{\circ}$ and $75^{\circ}$, in order to be close to the Brewster angle of $\mathrm{Si}$ for some wavelengths within the spectral range. The non-focused spot size has a diameter of around $2 \mathrm{~mm}$, and so a projected major axis between 4.7 and $7.7 \mathrm{~mm}$ (for angles of $65^{\circ}$ and $75^{\circ}$, respectively). The MIR measurements were performed with a Semilab IRSE equipment, which is a variable angle Fourier-transform infrared ellipsometer. This setup allows a spectral width of $600-7500 \mathrm{~cm}^{-1}$ $(1.3-16.7 \mu \mathrm{m})$ with a very high resolution of about $0.5 \mathrm{~cm}^{-1}$, when using a liquid nitrogen cooled $\mathrm{HgCdTe}$ detector. Due to the resolution capabilities, IRSE is ideal for the measurement of layers of several $10 \mu \mathrm{m}$ thickness, as it can resolve the interference oscillations in the ellipsometric spectra. Infrared illumination at an angle of incidence of $70^{\circ}$ through an aperture was chosen in our case to produce a spot size of $4 \mathrm{~mm}$, as it is a good compromise to minimize PSL thickness non-uniformity on the illuminated area, while having a more than adequate reflected light intensity.

The recorded ellipsometric spectra were evaluated with the Woollam CompleteEASE v5.04 and Semilab SEA v1.2.30 data analysis software products. These software use regression analysis to fit the free parameters of the optical models by the minimization of a well-defined merit of fit, in our case the mean squared error (MSE), defined in the following way:

$$
M S E=\sqrt{\frac{1}{n-P-1} \sum_{j=1}^{n}\left\{\left(X_{j}^{\text {meas }}-X_{j}^{\text {calc }}\right)^{2}\right\}},
$$

where $X^{\text {meas }}$ are the measured and $X^{\text {calc }}$, the calculated ellipsometric values, $n$ is the number of independently measured values, $P$ is the number of unknown model parameters. $X$ are either $N=\cos (2 \psi), C=\sin (2 \psi) \cos (\Delta)$ or $S=\sin (2 \psi) \sin (\Delta)$, when assuming isotropic samples for M-2000DI evaluations or $\alpha=$ $\cos (2 \psi)$ or $\beta=\sin (2 \psi) \cos (\Delta)$ for the IRSE evaluations. These values are linearly related to the measured intensity harmonics modulated by either a rotating compensator (for $N, C$ and $S$ ) or a rotating analyzer (for $\alpha$ and $\beta$ ) $[25,26]$, (Handbook of Ellipsometry, pp. 523-534 [27]). This is the reason why we define the MSE by these values instead of $\Psi$ and $\Delta$, and weighting every measurement point of the spectrum as unity, but for illustration 
purposes $\Psi$ and $\Delta$ are more convenient. Additionally, fitting has been made separately on the spectra of the two measurements (for the same sample) to avoid weighting of the ellipsometric values due to the conversion from either of the ranges and also because the illumination is not sure to be incident on the same spot on the samples.

\section{Optical model development}

Different optical models for ellipsometric evaluations were developed with increasing complexity. Porosity can be modeled with the help of the Bruggeman effective medium approximation [28], because the typical pore dimensions are much less $(<50 \mathrm{~nm})$ than the wavelength of the illuminating light, and so diffraction and non-specular scattering are negligible. The effective medium layers (EML) consisted of varying ratio of monocrystalline silicon (c-Si) and void with additional stoichiometric $\mathrm{SiO}_{2}$ present in the oxidized samples. The complex dielectric functions required for the description, the optical response of the semi-infinite substrate and the constituents of the EML were taken from the literature for c-Si in the UV-NIR, c-Si in the MIR and $\mathrm{SiO}_{2}$ in the UV-NIR and from reference measurements for $\mathrm{SiO}_{2}$ in the MIR range.

The PSL span across a large thickness range, and above around $5 \mu \mathrm{m}$ the interference oscillations in the ellipsometric spectra become too dense to be resolved by UV-NIR measurements. As a consequence, two modeling schemes are considered. For the samples with a nominal thickness smaller than $5 \mu \mathrm{m}(\mathrm{N}-01-\mathrm{N}-08)$ similar optical models can be used for the UVNIR and MIR spectra, that is, one or more EML describing the porosity on top of a semi-infinite c-Si substrate. On the other hand, for the thicker PSL (N-09-N-11), fitting is only done at the shorter wavelengths $(191-700 \mathrm{~nm})$ for the UV-NIR measurements, describing the surface and near-surface porosity with an EML and a semi-infinite effective medium, respectively, without sensing the bottom of the PSL due to the light absorption of the $\mathrm{c}-\mathrm{Si}$ (thus the unresolvable $700-1690 \mathrm{~nm}$ part of the spectra are excluded from the fitting).

For the MIR measurements of the thicker PSL, the part where the interferences can be well resolved (at longer wavelengths) is chosen for the fitting procedure, allowing a thickness and porosity evaluation similarly to the thinner samples. In Fig. 2, four different optical models are shown with increasing complexity. The top row represents the model structure, while the bottom row is a fit example, specifically the difference between fitted and measured $\Psi$ and $\Delta$ values for $\mathrm{N}-02(1.2 \mu \mathrm{m}$ thick PSL) measured at the UVNIR range at 3 different angles of incidence. The $P$ and MSE values are also shown. To describe porosity inhomogeneity in depth, multiple stacked EML were used. In case the sublayers are independently fitted, i.e. the thickness and void parameters are uncoupled between the different sublayers as shown in Fig. 2a, then we cannot increase the number of sublayers $(m)$ to more than 3 (model M1), because parameter cross-correlations become too high, making the fitted results questionable or even unphysical. Of course, by coupling the thickness values of the sublayers, we can slightly increase $m$ (one or two additional sublayers), but other simulation artifacts can also appear if independent EML are used to describe a continuous porosity gradient. In fact, simulations of a multi-EML structure, where a difference of porosity content more than a few percent between two neighboring independent sublayers is present, will cause additional small interference oscillations absent in the measurements (see Fig. 2a).

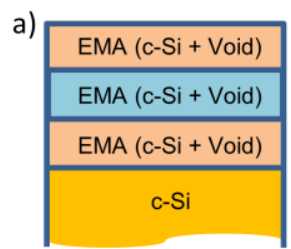

$M S E=33 \quad P=6$

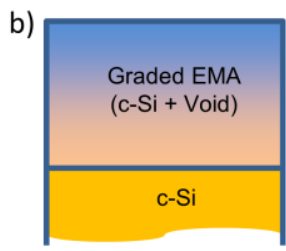

$M S E=38 \quad P=3$

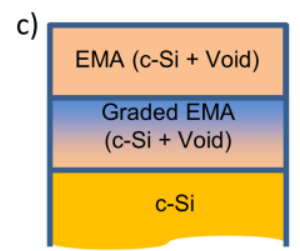

$M S E=14 \quad P=4$

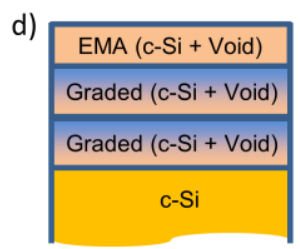

$M S E=11 \quad P=6$

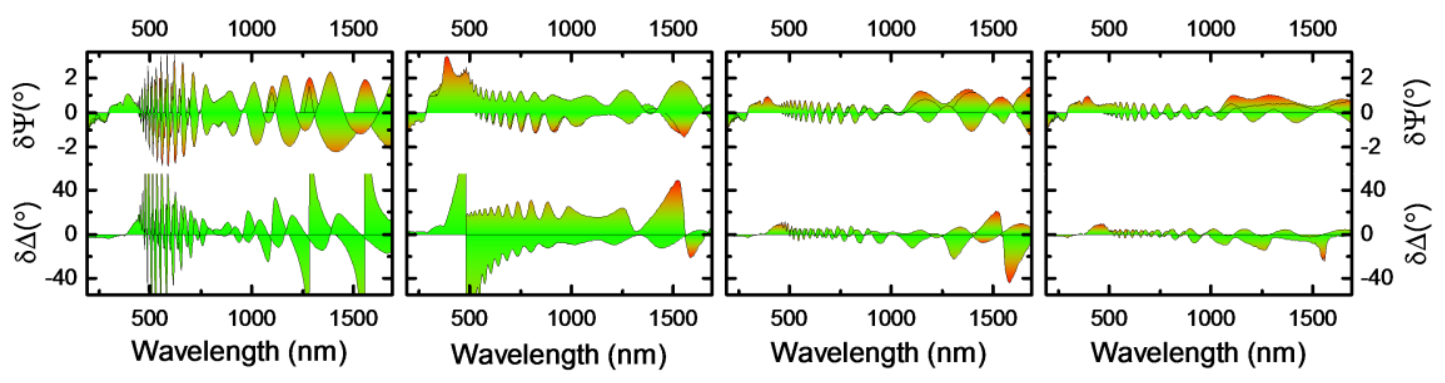

Figure 2: Ellipsometric models (top row) and difference (bottom row) between the measured and fitted $\psi$ and $\Delta$ values (for incident angles of $65^{\circ}, 70^{\circ}$ and $75^{\circ}$ ) of sample N-02 (1.2 $\left.\mathrm{m} \mathrm{PSL}\right)$. The difference spectra belonging to the different angles of incidence are plotted together as the area under the curves thus pointing out the largest difference at each wavelength. Three independent EML (model M1) as leftmost (a), graded EML (model M2) as middle left (b), graded EML with surface roughness (model M3) as middle right (c) and coupled double graded EML (model M4) as rightmost graph (d). Fit mean squared error (MSE) and number of fitted parameters $(P)$ are also shown.

An improved model consists of a graded EML structure; in this case, the in-depth porosity gradient is described by a simple linear function with 3 fitted parameters: global thickness of the PSL and void ratio at the top and the bottom of the PSL (model M2). As it can be seen from Fig. $2 b$, this model addresses the artificial oscillations seen in Fig. 2a, but a more global difference appears between the fitted and measured ellipsometric angles. Although there is a slight increase in the MSE values compared to those obtained with the previous model, the few number of fit parameters indicates that the simple linear description of the porosity gradient is a good basis for further model improvements. The reason why model $\mathrm{M} 2$ does not produce a merit of fit as good as in the case of model M1 is because with model M1 the surface roughness was indirectly included as the top $E M L$, which is not the case for model M2. If we include a surface roughness layer on top of the graded linear layer (model M3), we will have a very good merit of fit, as it is demonstrated in Fig. 2c. Usually, the surface roughness layer is defined as an EML composed of $50 \%$ void and $50 \%$ of the underlying layer, but in our case to better describe the unique surface of the PSL, no such fixation of the composition 
ratio has been made, i.e. a separate EML of $\mathrm{c}-\mathrm{Si}$ and void with fitted thickness and void ratio has been chosen.

A further improvement of the models is to consider an interface layer between the bottom of the PSL and the top of the substrate. For this purpose, a second graded EML structure was added as an interface in such a way that the top c-Si/void ratio of this layer is coupled to the bottom of the main graded EML structure and the bottom of this interface diminishes to zero void percent (model M4). The typical thickness values for the surface roughness (the top EML) are $10-20 \mathrm{~nm}$, for the bottom interface (the bottom EML) are 60-100 $\mathrm{nm}$, while the main graded EML gives up the rest of the PSL thickness for the samples thinner than $5 \mu \mathrm{m}(\mathrm{N}-01-\mathrm{N}-08)$. The infrared illumination is insensitive to the surface roughness layer and the bottom interface layer and so they have not been included in the MIR evaluations, the simpler M2 model has been used. The model M4 scheme and a relative fit example can be seen in Fig. $2 \mathrm{~d}$. As it can be seen from the MSE, there is an improvement in the fit quality compared to M3. If compared to $\mathrm{M} 1$ which has the same number of independent fit parameters, we obtain an increase in the fit quality by a factor of three. Thickness non-uniformities of the PSL had also been included in the models (model M5), which further improved the fit quality (slightly for samples $\mathrm{N}-01-\mathrm{N}-08$, more so for samples $\mathrm{N}$ $09-\mathrm{N}-11$ ). Anisotropic optical models were also tested. Fitting revealed that for the thinner PSL it had no, or negligible effect on fit quality, while for the thickest PSL, anisotropy of the layer has similar but only a mild effect on the evaluations as porosity depth inhomogeneity. Combined effects will be investigated in future studies with different measurement configurations.

It is important to note that because the ellipsometric models used for the simulation of the reflections consider optically homogeneous layers, any EML gradient described by a function must also be discretized into homogeneous sublayers. Because of computer CPU time considerations, choosing arbitrarily large number of sublayers is disadvised. To find the optimal $m$, we investigated how the fit quality depends on it (see Fig. 3 for samples $\mathrm{N}-01$ to $\mathrm{N}-04$ ). The MSE converges to a certain value, but the convergence degree depends on the thickness: for thinner PSL, the MSE will not improve with $m$ more than $20-30$ while for the thickest PSL, 100 sublayers are preferred for the best fit.

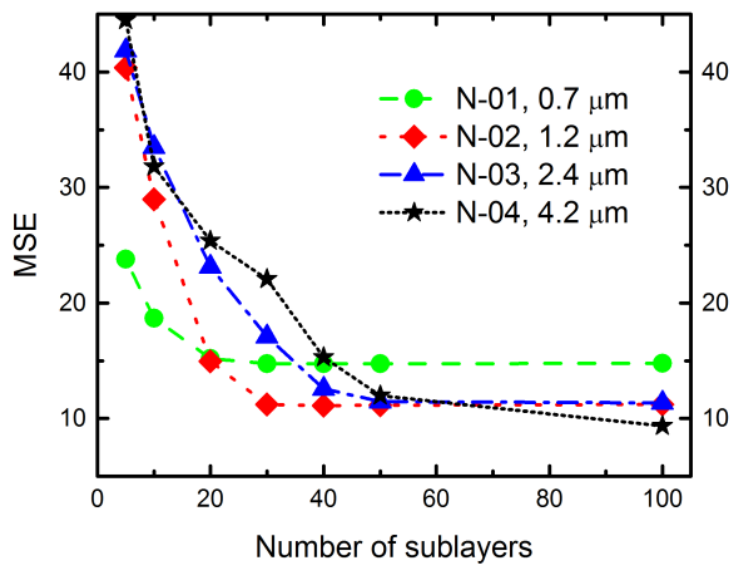

Figure 3: Mean squared error (MSE) dependency as a function of the number of EML sublayers used in model M5 for fitting of four PSL with different thickness (samples $\mathrm{N}-01$ to $\mathrm{N}-04$ ).

As mentioned in the introduction, the oxide content within the pores cannot be sensitively quantified with UV-NIR measurements, because it strongly correlates with the void content. This limitation can be surpassed by fitting the MIR measurements due to the characteristic line-shape of the refractive index $(n)$ and extinction coefficient $(k)$ of $\mathrm{SiO}_{2}$ at around a wavenumber of $1100 \mathrm{~cm}^{-1}$. To this end, the two-component EML was supplemented by an additional $\mathrm{SiO}_{2}$ component (model M6) of which the $n$ and $k$ optical parameters were obtained from reference thin layer measurements.

In Fig. 4, the effect of such $\mathrm{SiO}_{2}$ content is demonstrated with $20 \mu \mathrm{m}$ thick PSL simulation. The top graph shows the $\psi$ and $\Delta$ values in a narrow spectral range at an angle of incidence of $70^{\circ}$ for PSL composed of $40 \%$ void and $0 \% \mathrm{SiO}_{2}$, while the bottom graph simulates the same PSL but with $20 \%$ void and $20 \% \mathrm{SiO}_{2}$. The characteristic optical properties of $\mathrm{SiO}_{2}$ at these wavelengths (see middle graph in Fig. 4) have a huge effect on the ellipsometric spectra, which makes the oxide content sensitively quantifiable. Although light absorption of $\mathrm{EML}$ with large $\mathrm{SiO}_{2}$ content at peak values of $k$ results in an optical penetration depth (OPD) of micrometer extent depending on the $\mathrm{SiO}_{2}$ quantity, the rest of the spectra with lower $k$ (and higher OPD) around the peak is enough to determine the average $\mathrm{SiO}_{2}$ volume fraction.

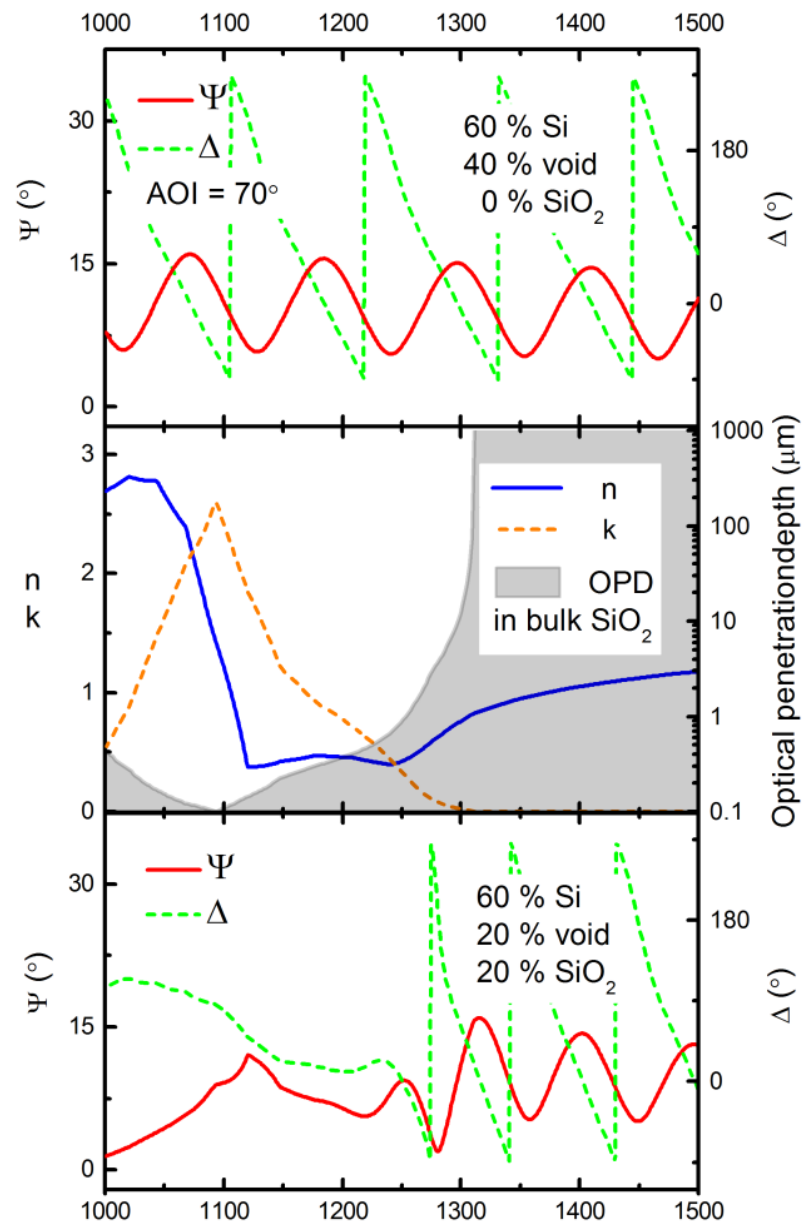

Wavenumber $\left(\mathrm{cm}^{-1}\right)$

Figure 4: Comparison of a simulation of a $20 \mu \mathrm{m}$ thick effective medium layer with $60 \% \mathrm{Si}, 40 \%$ void and $0 \% \mathrm{SiO}_{2}$ (top layer) and of an equally thick effective medium layer but with $20 \%$ void and $20 \% \mathrm{SiO}_{2}$ (bottom layer). The effect of the optical properties ( $\mathrm{n}-$ refractive index, $\mathrm{k}$ - absorption coefficient and OPD - optical penetration depth) of the $\mathrm{SiO}_{2}$ (middle layer) produces a very distinct difference in the simulations around $1100 \mathrm{~cm}^{-1}$.

OPD is also shown for pure $\mathrm{SiO}_{2}$ in the middle graph in Fig. 4. These values could be higher by several factors in a mixed EML, and ellipsometric depth sensitivity is typically three times larger than OPD (see Fig. 4 in Ref. [29]). Depth profiling $\left(\mathrm{SiO}_{2}\right.$ vertical inhomogeneity characterization) remains difficult, because of the narrow $\mathrm{SiO}_{2}$ sensitive part of the spectra that can probe the entire layer. 


\section{Evaluation results}

From the optical models of M5 for UV-NIR and M6 for MIR, evaluations have been made for all the samples with a nominal PSL thickness smaller than $5 \mu$ m (i.e. for samples $\mathrm{N}-01$ to $\mathrm{N}-08$ ). For example Fig. 5a (MIR) and Fig. 5b (UV-NIR) spectra represent the measured and fitted $\psi$ and $\Delta$ values for sample N-06 $(1.2 \mu \mathrm{m}$ thick). It can be seen that the fit is excellent throughout the whole spectra for both wavelength range (MSE = 14 for UV-NIR fit and $M S E=11.9$ for MIR fit). Similar excellent fits can be made on the other thin samples. Fig. 6 represents the evaluated thickness values plotted versus the consumed charge density. Results are averaged from the optical and the infrared SE measurements, with a typical difference of 3-4 \% between the two methods. A clear linearity can be observed, suggesting that, when restricted to this range, the current density has a limited impact on the etch rate of thin PSL and so, on dissolution valence. The fitted ellipsometric thickness values also correlate well with SEM measurements (Table 1). a)

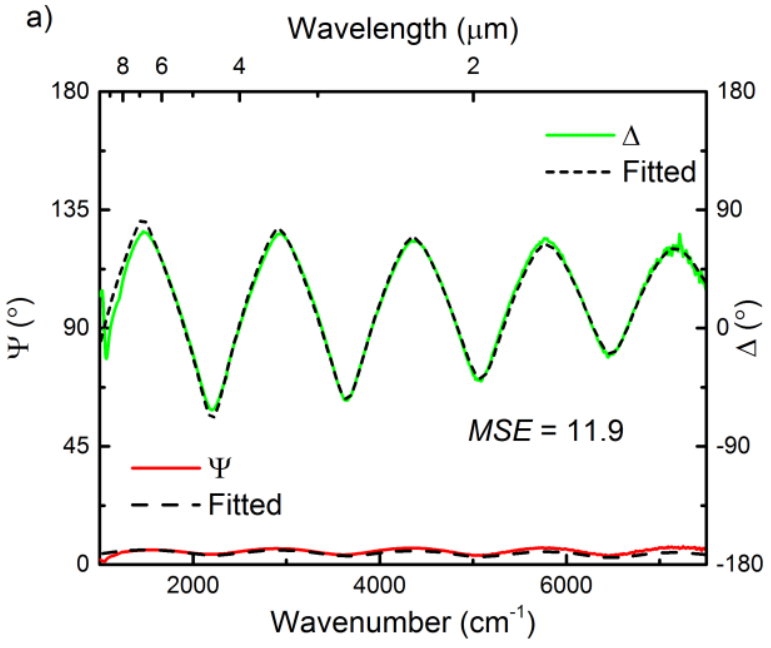

c)

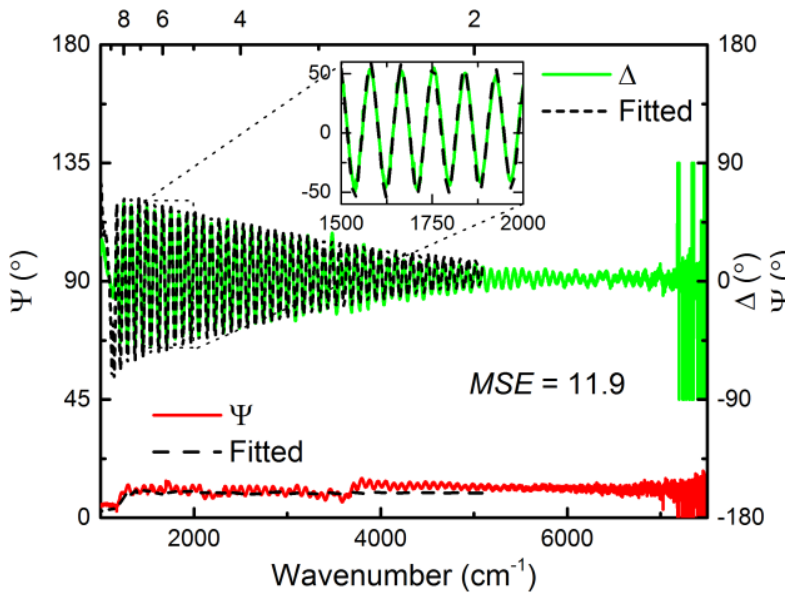

b)

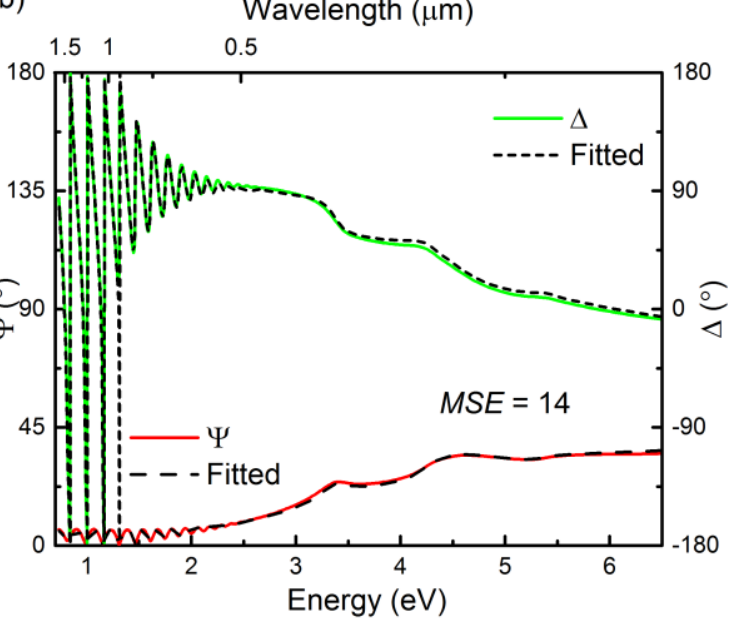

d)

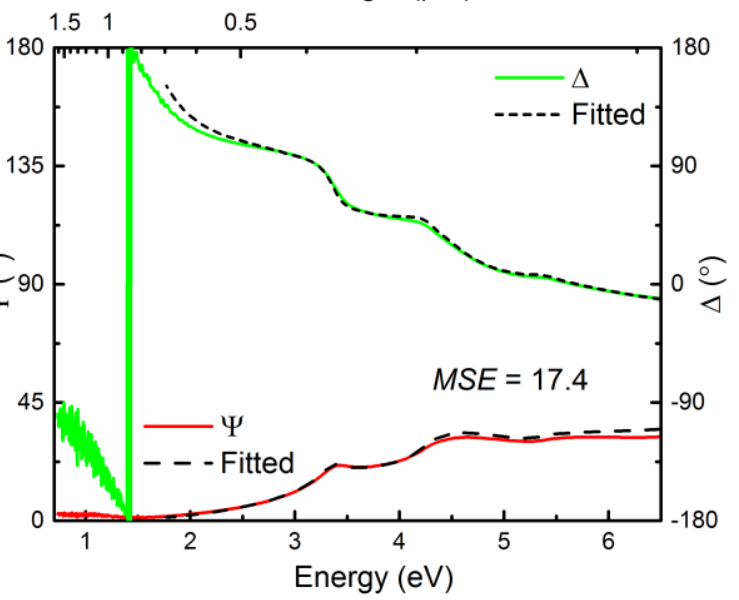

Figure 5: Measured and fitted ellipsometric angles of a less than $5 \mu \mathrm{m}$ thick PSL (sample N-06, $1.2 \mu \mathrm{m}$ thick) in the MIR wavelength range (a), and in the UV-NIR wavelength range (b) and of a more than $5 \mu \mathrm{m}$ thick PSL (sample N-09, $23 \mu \mathrm{m}$ thick) in the MIR wavelength range (c), and in the UV-NIR wavelength range (d). 


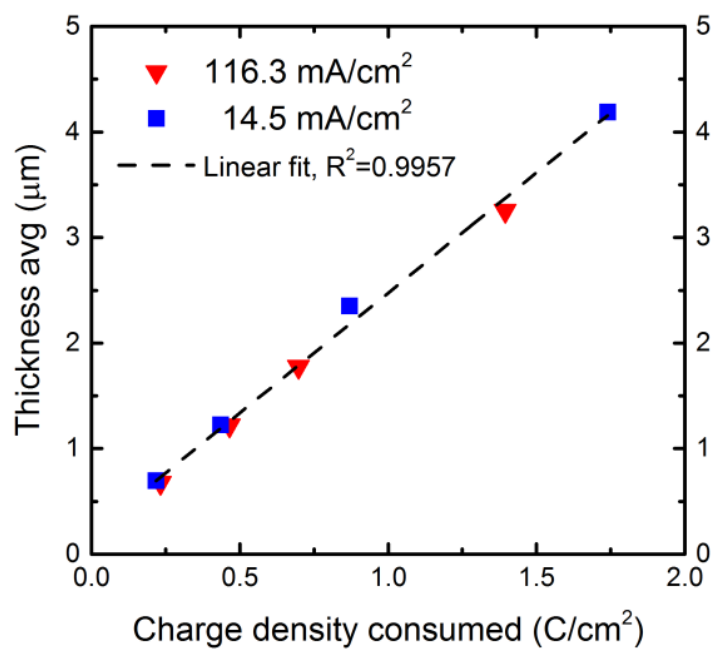

Figure 6: Evaluated thickness values for the injected charge density consumed during anodization for samples obtained at $14.5 \mathrm{~mA} / \mathrm{cm}^{2}$ and the ones obtained at $116.3 \mathrm{~mA} / \mathrm{cm}^{2}$ (samples $\mathrm{N}$ 01 to $\mathrm{N}-08$ ). The results are averaged from the optical and the infrared SE measurements.

In Fig. 7, porosities of the thin PSL are shown as a function of the layer thickness. For the UV-NIR measurements fitted by model M5, the in-depth average values of the doubly graded layers are shown, while for the MIR measurements, the plotted porosities are the sum of the fitted void and $\mathrm{SiO}_{2}$ volume fractions from model M6. Firstly, it is important to notice that similar results were extracted from either spectra appertaining to the two ellipsometers. Then, no definite characteristic tendencies can be observed; rather, porosities are constant within $6 \%$, although higher current densities typically produce slightly higher porosities for similar layer thicknesses [30]. The relative thinness of the porous layers produced in this study may explain such limited variations in porosity with depth.

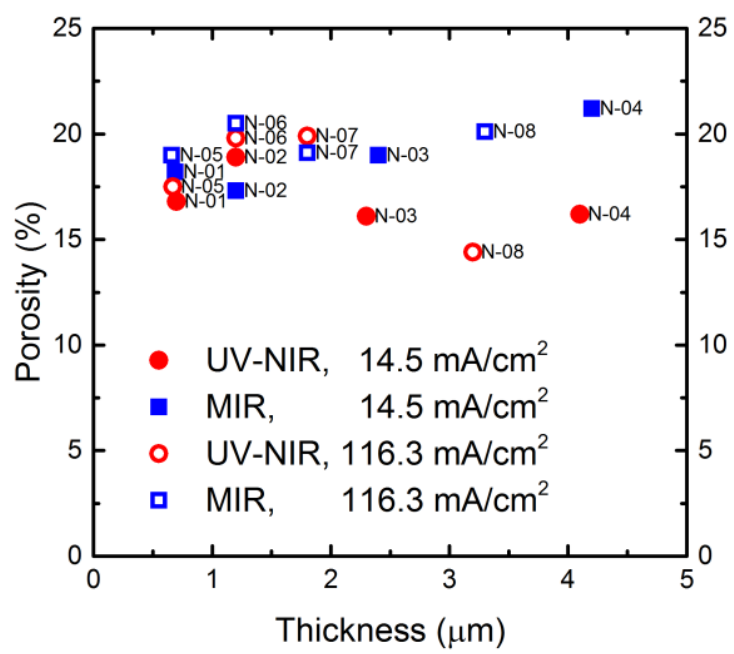

Figure 7: Fitted average in-depth porosity values, i.e. void volume fractions for the UV-NIR and sum of void and $\mathrm{SiO}_{2}$ volume fractions for the MIR measurement in function of the fitted thickness values evaluated with models $\mathrm{M} 5$ and $\mathrm{M} 6$, respectively, of the less than $5 \mu \mathrm{m}$ thick PSL (samples $\mathrm{N}-01$ to $\mathrm{N}-08$ ).

As for the thicker PSL, an example of fitted spectra (that of the $23 \mu \mathrm{m}$ PSL, N-09) is demonstrated in Figs. 5c (MIR) and 5d (UVNIR). The fits on the MIR spectra are good for wavelengths above
$2 \mu \mathrm{m}$ (below $\sim 5000 \mathrm{~cm}^{-1}$ ). For the remaining part of the wavelength range, the measurements are too noisy and the thickness non-uniformity smears the clear oscillations. Furthermore, fits on the UV-NIR spectra are good for wavelengths below $700 \mathrm{~nm}$ (above $1.8 \mathrm{eV}$ ). At this wavelength range, there is no reflection from the silicon/porous silicon interface, because the OPD of light in the PSL is much smaller than the layer thickness. Above $700 \mathrm{~nm}$, layer interference oscillations appear, but they are too dense to be resolved and thus fitted.

Porosity results are shown in Fig. 8 for the thickest samples ( $N$ 09 to $\mathrm{N}-11$ ). Surface porosity from the UV-NIR evaluations and the average porosity and oxide content from the MIR evaluations are shown. Average porosity increases rapidly with thickness, while surface porosities increases slowly. The rise of average porosity with anodization duration was previously observed on both highly-doped $p$ - and n-type silicon [31-33], (Handbook of Porous Silicon, pp. 11-22 [34]). This phenomenon is generally attributed to a diffusion-limited renewal of the electrolyte at the silicon/porous silicon interface during long-duration anodizations. A progressive decrease in $\mathrm{HF}$ concentration at the reactive interface implies a simultaneous increase of the porosity [30]. Electrolyte exhaustion is all the more visible when the sample is thick, which explains why this tendency is only observable between the $\mathrm{N}-09, \mathrm{~N}-10$ and $\mathrm{N}-11$ samples. As for surface porosity, its slight increase with anodization duration can mainly be explained by a pure chemical etching phenomenon of silicon by HF [34].

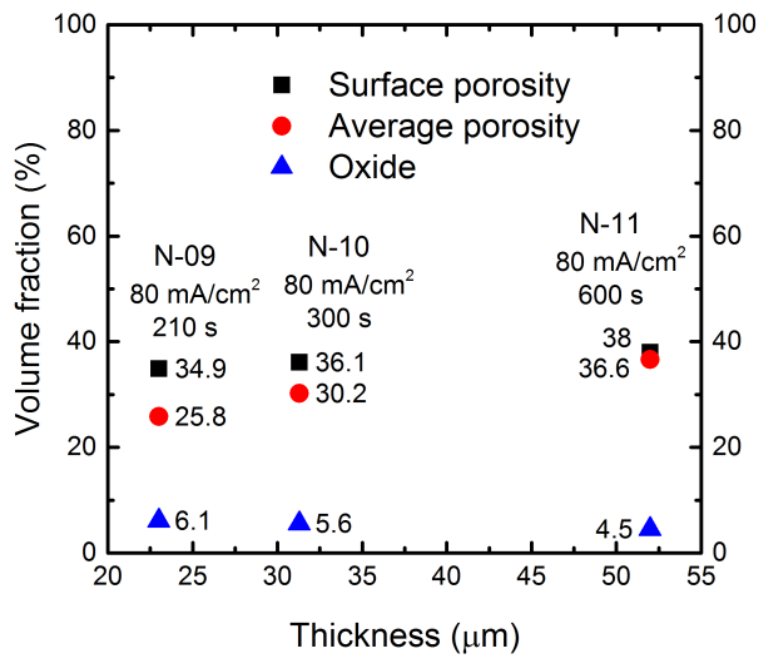

Figure 8: Surface porosity from UV-NIR evaluations and the average porosity and oxide content from MIR evaluations represented by volume fractions as a function of the thickness for $\mathrm{N}-09$ to $\mathrm{N}-11$ samples.

Concerning the evaluations of the oxidized porous samples, with the help of model M6, the different volumetric contents (Si, void and $\mathrm{SiO}_{2}$ components) could be obtained, as shown in Fig. 9. After being anodized (Ox-Ref), the oxide content of the PSL is less than $1 \%$. However, a low-temperature oxidation at $300^{\circ} \mathrm{C}$ is sufficient to increase the oxide content from $0.9 \%$ to $15 \%$ (Ox-1), indicating back-bond oxidation [35] in which oxygen atoms are inserted between silicon - silicon hydrides $\left(\mathrm{Si}_{-} \mathrm{SiH}_{\mathrm{x}}\right)$ bonds. 
$\square \mathrm{Si} \square$ Void $\square \mathrm{S} \mathrm{SiO}_{2}$

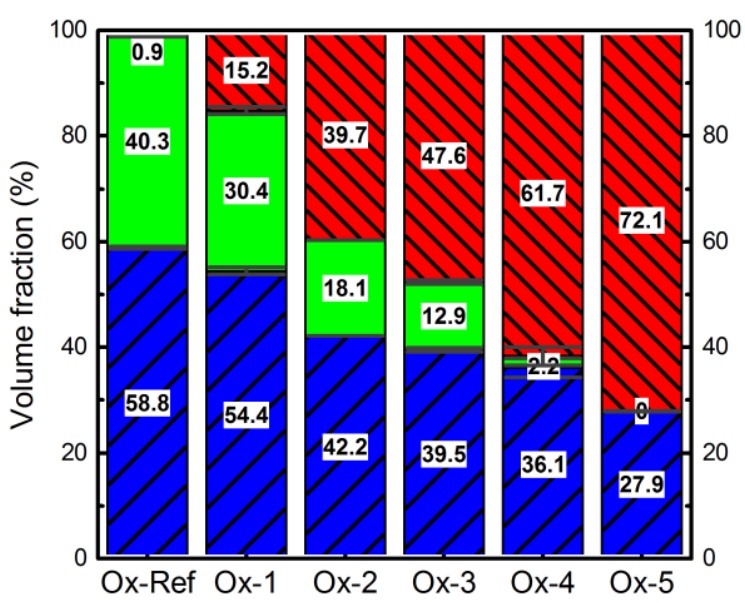

Figure 9: Volume fraction percent of the $\mathrm{Si}$, void and $\mathrm{SiO}_{2}$ contents for the different oxidized porous samples with error bars for $\mathrm{Si}$ and void in dark gray obtained by the difference between fitted and expected values. The expected values are calculated from the initial porosity and the measured $\mathrm{SiO}_{2}$ volume content by taking into account the $44 / 56$ ratio of volumetric expansion when oxidizing $\mathrm{Si}$, (see eq. $2 \mathrm{a}$ and $2 \mathrm{~b}$ ).

Thermally oxidized samples $0 x-4$ and $0 x-5$ exhibit a high oxide content in the structure in which the void content tends to 0 $\%$ with the oxidation duration. Anodically oxidized samples show a lower oxide content than thermally oxidized ones. The oxide content of sample Ox-3, combining successively electrochemical and thermal oxidation, is surprisingly lower than the one of sample Ox-5, only subjected to thermal oxidation. This result may suggest a pore-closing on top of the layer preventing oxygen diffusion to the remaining silicon. This hypothesis is confirmed by the significant fraction of remaining void in the electrochemically oxidized PSL (almost 13\%).

Volumetric expansion occurs during oxidation as $\mathrm{SiO}_{2}$ consumes $\mathrm{Si}$ to grow. In case of a flat silicon wafer, $44 \%$ of $\mathrm{SiO}_{2}$ total thickness is grown under the initial level of silicon. As a result, if the original porosity without any oxide is known, we can calculate an expected $\mathrm{Si}$ and void volume content from the measured $\mathrm{SiO}_{2}$ volume fractions. In other words, the $\mathrm{Si}, \mathrm{SiO}_{2}$ and void contents are not independent from each other during the porous framework oxidation; the following equations describe their relation:

$$
\begin{aligned}
& V_{S i}=1-V_{i p}-0.44 V_{S i O 2} \\
& V_{\text {void }}=V_{i p}-0.56 V_{\text {SiO } 2}
\end{aligned}
$$

where, $V_{S i}, V_{S i O 2}$ and $V_{\text {void }}$ are the volume fractions of $\mathrm{Si}, \mathrm{SiO}_{2}$ and voids respectively, while $V_{i p}$ is the initial porosity before oxidation $[10,36]$. We could have implemented these equations in the fitting procedure coupling these parameters together and reducing the overall number of fit parameters, but in our case it was fitted independently to verify the difference between the fitted and empirically calculated values afterwards.

In Fig. 10 we can see such an implementation, that is, the strong correlation between independently measured and expected (calculated from $V_{i p}$ and $V_{S i_{2}}$ ) volume fractions. $V_{i p}$ could be chosen as the void volume fraction of the reference measurement, but a small, probably native oxide is already present $(0.9 \%)$ in the PSL, thus biasing the equations. To correct the volume fraction values of $\mathrm{Si}$ and void, the $V_{\mathrm{SIO}_{2}}$ has been extrapolated to 0 and $V_{i p}$, fitted in such a way as to minimize the measured and calculated $V_{S i}$ and $V_{\text {void }}$. The error values plotted in Fig. 9 are the differences between the measured and expected values explained above (the errors from the fit of model M6 are smaller, less than $1 \%$, not shown in the figure). Consequently, as the error values are rather small, the validity of model M6 and of the fitted volume fractions are reinforced. Moreover, the validity of the model is not influenced by the type of oxide. Indeed, the oxide obtained by electrochemical oxidation can also be characterized accurately. The fit on the oxide content is coarsely based on the Si-O-Si absorption peak around $1100 \mathrm{~cm}^{-1}$, thus making this technique robust and largely independent of the oxidation method.

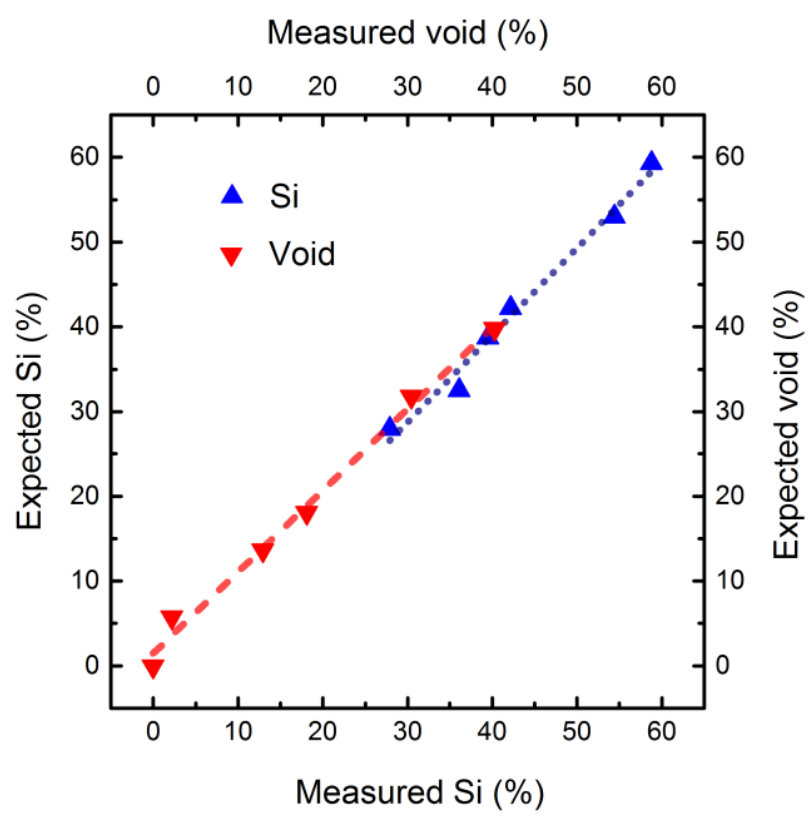

Figure 10: Correlation between the independently measured and expected volume fractions (see eqs. $2 a$ and $2 b$ for the calculation of the expected values).

\section{Conclusions}

Investigation of the PSL with optical and infrared ellipsometers allowed analyses in a broad spectral range, and thus the possibility to determine both the thickness (for over two orders of magnitude) and the porosity distribution of the PSL. Several different optical models were developed with increasing complexity and tested to describe the porosity content of the PSL, such as the multiple independent EML (model M1), graded EML (model M2), graded EML with surface roughness (model M3), coupled double-graded EML with surface roughness (model M4), and M4 with thickness non-uniformity (model M5). The best model was chosen in a way to minimize the MSE while keeping the fitting error and the parameter cross-correlations low. As for UV-NIR ellipsometry, model M5 takes into account the thickness inhomogeneity, surface roughness, pore (2b) initiation, in-depth porosity gradient and the silicon/porous silicon interface. UV-NIR ellipsometry is sensitive to surface and near surface characterizations without adequate optical separation of oxide and porosity values, while the MIR ellipsometry, being sensitive to the absorption peak of $\mathrm{SiO}_{2}$, can resolve the oxide content by adding a third phase in the EML (model M6). Thin PSL shows no impact of current density on porosity and thickness. For thick samples, evaluation results highlight the in-depth porosity gradient when the anodization duration exceeds a few minutes, whereas surface porosity only slightly increases. Finally, oxidation techniques have been compared in terms of oxidation level and void content. Volume expansion during PSL oxidation follows exactly the same behavior as that during the oxidation of planar silicon wafers. With the appropriate optical model development, ellipsometry proves to be one of the most powerful techniques to swiftly and accurately evaluate PSL characteristics. 


\section{Acknowledgments}

This work was supported by the National Development Agency grants SROP-4.2.2/B-10/1-2010-0029, SROP-4.2.2.B-10/1-20100025, by OTKA grant Nr. K81842, by ENIAC E450EDL project and by the Hungarian-French Intergovernmental S\&T Cooperation Programme grant Nr. TÉT_12_FR-1-2013-0016 and by CampusFrance in the frame of a Balaton PHC project. We also thank Semilab Semiconductor Physics Laboratory for allowing access to IRSE and for their supporting knowledge.

\section{References}

[1] M.S. Salem, M.J. Sailor, F. a. Harraz, T. Sakka, Y.H. Ogata, J. Appl. Phys. 100 (2006) 83520. doi: 10.1063/1.2360389

[2] E. Luais, F. Ghamouss, J. Wolfman, S. Desplobain, G. Gautier, F. TranVan, J. Sakai, J. Power Sources 274 (2015) 693. doi: 10.1016/j.jpowsour.2014.10.084

[3] M. Capelle, J. Billoué, P. Poveda, G. Gautier, Electron Devices, IEEE Trans. 58 (2011) 4111. doi: 10.1109/TED.2011.2164078

[4] G. Gautier, P. Leduc, Appl. Phys. Rev. 1 (2014). doi: 10.1063/1.4833575

[5] L. Canham, in:, Handb. Porous Silicon, 2014, pp. 1-6. doi: 10.1007/9783-319-04508-5 19-1

[6] A. Loni, T. Defforge, E. Caffull, G. Gautier, L.T. Canham, Microporous Mesoporous Mater. (2015) 10. doi: 10.1016/j.micromeso.2015.03.006

[7] M.J. Sailor, Porous Silicon in Practice: Preparation, Characterization and Applications, Wiley. com, 2012. doi: 10.1002/9783527641901

[8] N. Ennejah, S. Aouida, B. Bessais, Phys. Status Solidi 8 (2011) 1931. doi: 10.1002/pssc. 201000083

[9] V. Chamard, P. Bastie, D. Le Bolloch, G. Dolino, E. Elkaïm, C. Ferrero, J.P. Lauriat, F. Rieutord, D. Thiaudière, Phys. Rev. B 64 (2001) 245416. doi: 10.1103/PhysRevB.64.245416

[10] S. a. Alekseev, V. Lysenko, V.N. Zaitsev, D. Barbier, J. Phys. Chem. C 111 (2007) 15217. doi: 10.1021/jp0712452

[11] G. Desplobain, S., Ventura, L., Defforge, T., \& Gautier, Porous Semicond. - Sci. Technol. (2014). http://www.thepsst.com/index.php?conference=psst\&schedConf=2014\&page=pap er\&op=viewPaper\&path[] $=229$

[12] E. Segal, L. a. Perelman, F. Cunin, F. Di Renzo, J.-M. Devoisselle, Y.Y. Li, M.J. Sailor, Adv. Funct. Mater. 17 (2007) 1153. doi: 10.1002/adfm.200601077

[13] M. Fried, T. Lohner, P. Petrik, in:, H.S. Nalwa (Ed.), Handb. Surfaces Interfaces Mater. Solid Thin Film. Layers, Academic Press, San Diego, 2001.

[14] A. Shan, M. Fried, G. Juhasz, C. Major, O. Polgar, A. Nemeth, P. Petrik, L.R. Dahal, J. Chen, Z. Huang, N.J. Podraza, R.W. Collins, IEEE J. Photovoltaics 4 (2014) 355. doi: 10.1109/JPHOTOV.2013.2284380

[15] P. Petrik, É. Vázsonyi, M. Fried, J. Volk, G.T. Andrews, a. L. Tóth, C.S. Daróczi, I. Bársony, J. Gyulai, Phys. Status Solidi C Conf. 2 (2005) 3319. doi: 10.1002/pssc. 200461153

[16] P. Petrik, M. Fried, E. Vazsonyi, P. Basa, T. Lohner, P. Kozma, Z. Makkai, J. Appl. Phys. 105 (2009) 1. doi: 10.1063/1.3068479
[17] E. Agocs, P. Petrik, S. Milita, L. Vanzetti, S. Gardelis, a. G. Nassiopoulou, G. Pucker, R. Balboni, M. Fried, Thin Solid Films 519 (2011) 3002. doi: 10.1063/1.3068479

[18] M. Fried, H. Wormeester, E. Zoethout, T. Lohner, O. Polgár, I. Bársony, Thin Solid Films 313-314 (1998) 459. doi: 10.1016/S0040 6090(97)00864-X

[19] M. Fried, O. Polgár, T. Lohner, S. Strehlke, C. Levy-Clement, J. Lumin. 80 (1999) 147. doi: 10.1016/S0022-2313(98)00086-6

[20] T. Lohner, M. Fried, P. Petrik, O. Polgár, J. Gyulai, W. Lehnert, Mater. Sci. Eng. B Solid-State Mater. Adv. Technol. 69 (2000) 182. doi: 10.1016/S0921-5107(99)00257-3

[21] R. Kitamura, L. Pilon, M. Jonasz, Appl. Opt. 46 (2007) 8118. doi: 10.1364/AO.46.008118

[22] K.S.W. Sing, D.H. Everett, R. a. W. Haul, L. Moscou, R. a. Pierotti, J. Rouquérol, T. Siemieniewska, Pure Appl. Chem. 57 (1985) 603. doi 10.1351/pac198557040603

[23] Michael J. Sailor, in: L. Canham (Ed.), Handb. Porous Silicon, Springer International Publishing, 2014, pp. 1-24. doi: 10.1007/978-3-31904508-5_37-1

[24] K.B. Clark, J. a. Bardwell, J.-M. Baribeau, J. Appl. Phys. 76 (1994) 3114. doi: $10.1063 / 1.357493$

[25] G.E. Jellison, Thin Solid Films 290-291 (1996) 40. doi: 10.1016/S0040 6090(96)09009-8

[26] H. Fujiwara, Spectroscopic Ellipsometry: Principles and Applications, John Wiley \& Sons Ltd., 2007. doi: 10.1002/9780470060193

[27] R. W. Collins, I. An, J. Lee, and J. A. Zapien, in: H. G. Tompkins, E. A. Irene, Hanbook of Ellipsometry, William Andrew, Inc., 2005, pp. 523-534.

[28] M. Fried, T. Lohner, O. Polgár, P. Petrik, É. Vázsonyi, I. Bársony, J.. Piel, J.. Stehle, Thin Solid Films 276 (1996) 223. doi: 10.1016/00406090(95)08058-9

[29] P. Petrik, E. Agocs, J. Volk, I. Lukacs, B. Fodor, P. Kozma, T. Lohner, S. Oh, Y. Wakayama, T. Nagata, M. Fried, Thin Solid Films (2014). doi: 10.1016/j.tsf.2014.02.008

[30] V. Lehmann, R. Stengl, a Luigart, Mater. Sci. Eng. B 69 (2000) 11. doi: 10.1016/S0921-5107(99)00286-X

[31] R. Herino, G. Bomchil, K. Barla, C. Bertrand, J.L. Ginoux, J. Electrochem. Soc. 134 (1987) 1994. doi: 10.1149/1.2100805

[32] S.E. Foss, P.Y.Y. Kan, T.G. Finstad, J. Appl. Phys. 97 (2005) 114909. doi: 10.1063/1.1925762

[33] T.D. James, a. J. Keating, G. Parish, C. a. Musca, J. Electrochem. Soc. 156 (2009) H744. doi: 10.1149/1.3174319

[34] A. Loni, in:, L. Canham (Ed.), Handb. Porous Silicon, Springer International Publishing, 2014, pp. 11-22. doi: 10.1007/978-3-319 05744-6_2

[35] Y.H. Ogata, T. Tsuboi, T. Sakka, S. Naito, 66 (2000) 63. doi: 10.1023/A:1009694608199

[36] K. Barla, R. Herino, G. Bomchil, J. Appl. Phys. 59 (1986) 439. doi: 10.1063/1.337036 